\title{
OBSERVATÓRIO NACIONAL DA DENGUE - SISTEMA PARA MONITORAMENTO DE CASOS DE DENGUE
}

\author{
Daniele Montenegro da Silva Barros \\ Instituto Federal de Educação, Ciência e Tecnologia do Rio Grande do Norte, Diretoria \\ Acadêmica de Gestão e Informática. monte.daniele@gmail.com \\ Philippi Sedir Grilo de Morais \\ Instituto Federal de Educação, Ciência e Tecnologia do Rio Grande do Norte, Diretoria \\ Acadêmica de Gestão e Informática. philippi.sedir@gmail.com \\ Jailton Carlos de Paiva \\ Universidade Federal do Rio Grande do Norte. jailtoncarlos@gmail.com \\ Jalerson Raposo Ferreira de Lima \\ Professor do Instituto Federal de Educação, Ciência e Tecnologia do Rio Grande do Norte, \\ Av. José Rodrigues de Aquino Filho, Nº 640, RN 120, Alto de Santa Luzia, Nova Cruz- \\ RN.jalerson@gmail.com \\ Janaína Luana Rodrigues da Silva \\ Bacharel em Secretaria Executivo, atualmente é pesquisadora do Laboratório de Inovação \\ Tecnológica em Saúde - HUOL/UFRN. Desenvolve pesquisa na área de Tele-trabalho \\ aplicado a saúde. janaina.lrsv@gmail.com
}

\section{RESUMO}

A dengue é um dos principais problemas de saúde pública no mundo, várias ações têm sido tomadas ao longo dos anos no combate a essa doença, e a utilização da Tecnologia da Informação pode ser uma grande aliada para alcançar esse objetivo. Dentro deste contexto, o Projeto Observatório Nacional da Dengue está inserido dentro das ações do Projeto Telessaúde, tendo por objetivo monitorar e ajudar a população e o Gestor Público no Combate à Dengue. Sendo assim, o presente trabalho tem por objetivo desenvolver um sistema que possibilite o monitoramento dos focos da dengue em tempo real, e como também, oferecer uma ferramenta que agilize o trabalho de campo do Agente de Endemias. Para a realização desse projeto foi necessário projetar uma arquitetura que possibilitasse a comunicação entre as informações coletadas pelo dispositivo móvel, o portal e o servidor, que é responsável pelo recebimento das informações.

PALAVRAS-CHAVE: Dengue, Dispositivo Móvel, Portal Web, Sistema Distribuído.

\section{NATIONAL OBSERVATORY OF DENGUE - SYSTEM FOR MONITORING OF CASES OF DENGUE}

\begin{abstract}
Dengue is a major public health problem in the world, several measures have been taken over the years to combat this disease, and the use of information technology can be a great ally to achieve this goal. Within this context, the Observatorio Nacional da Dengue is inserted into the actions of the Telehealth Project, aiming to monitor and assist the population and the Public Manager in effort to Combat Dengue. Therefore, this study aims to develop a system enabling the monitoring of dengue outbreaks in real time, but also
\end{abstract}


offer a tool to speed up the fieldwork of the Endemics Agent. For the realization of this project it was necessary to design an architecture that would enable communication between the information collected by the mobile device, the web portal, and the server, which is responsible for receiving the information.

KEYWORDS: Dengue, Mobile Device, Web Portal, Distributed System.

\section{OBSERVATÓRIO NACIONAL DA DENGUE - SISTEMA PARA MONITORAMENTO DE CASOS DE DENGUE}

\section{INTRODUÇÃO}

A informática é utilizada cada dia mais em diversas areas do conhecimento, estando presente no cotidiano das pessoas, mesmo quando elas não percebem, confirmando assim a natureza multidisciplinar e estratégica dessa area. Na saúde não seria diferente. A informática em saúde é uma linha de pesquisa que lida com recursos, dispositivos e métodos para otimizar o armazenamento, a recuperação e o gerenciamento das informações em saúde (SBIS, 2013).

Neste contexto, as ações voltadas para Atenção Básica em Saúde tem se intensificado nos últimos anos. Essa prática da medicina pode ser definida como ações individuais e coletivas voltadas à promoção da saúde, prevenção de agravos, tratamento e reabilitação (GIOVANELLA, 2009). Essas ações buscam atingir a população em geral confirmando assim, a sua natureza estratégica, politica e social. Nesse sentido, as ações de combate a dengue se enquadram dentro das politicas de Atenção Básica a Saúde, pois tem por objetivo atender as necessidades da população, exigindo o entendimento da saúde como direito social.

Um estudo realizado em oito países, incluindo o Brasil, mostrou que o custo com as epidemias de dengue geram uma despesa de aproximadamente U\$ 1,8 bilhão, somente com despesas ambulatoriais e hospitalares, sem incluir os custos com as atividades de vigilância, controle de vetores e mobilização da população. (DIRETRIZES NACIONAIS PARA A PREVENÇÃO E CONTROLE DE EPIDEMIA DE DENGUE, 2009).

Esse estudo comprova que a dengue é um problema de saúde publica. Nesse sentido, o governo federal mantem o programa de controle de zoonoses, e uma das ações desse programa, são atividades de controle da dengue, as quais visam combater a proliferação do vetor Aedes Aegypti, pois é por meio da picada do mosquito fêmea que ocorre a transmissão do vírus. O controle tem início através das operações de campo, na qual o Agente de Endemias posse de formulário de levantamento de índice, coleta informações com o objetivo de permitir o diagnóstico rápido da situação entomológica e assim auxiliar no direcionamento das ações de controle vetorial e de educação em saúde.

O problema é que esse levantamento é feito totalmente manual, os dados coletados pelos Agentes de Endemias, são passados pelo Encarregado de Combate a Endemias, o qual sintetiza as informações em formulários específicos e repassa para a Gerência de Controle de Zoonoses, que por sua vez repassa para a Secretária Municipal de Saúde, a qual irá processar as informações para gerar dados estatísticos. Toda essa operação ocasiona bastante tempo, em média dois meses, e como consequência a ocorrência erros de avaliação, uma vez que as áreas pesquisadas no início poderiam, ao longo desses dois 
meses, apresentarem mudanças no perfil da infestação vetorial. Dentro deste contexto, há a necessidade de ações e uso de ferramentas mais eficazes no sentido de agilizar o processo entre a coleta da informação sobre o foco, durante a realização do trabalho de campo, e a divulgação desse resultado para a população.

Neste sentido, o presente trabalho propõe desenvolver uma solução que visa munir a Secretária Municipal de Saúde com ferramental tecnológico para apoiar as ações de controle da dengue, favorecendo agilidade na obtenção dos resultados das pesquisas, facilitando o acompanhamento e avaliação dos trabalhos desenvolvidos. A solução é composta por dispositivos móveis para apoiar as operações de campo dos Agentes de Endemias, um portal web para apoiar as decisões das ações de controle vetorial, bem como divulgar para a população informações úteis sobre a dengue.

\section{DENGUE}

A dengue é um dos principais problemas de saúde pública no mundo. A Organização Mundial da Saúde (OMS) estima que 2,5 bilhões de pessoas o que representa 2/5 da população mundial, estão sobre risco de contrair dengue. Desse total, cerca de 550 mil necessitam de hospitalização e pelo menos 20 mil morrem em consequência da doença. No Brasil os primeiros registros da doença datam do início do século XIX, afetando principalmente as populações urbanas menos favorecidas e escravos rurais. No entanto, a primeira epidemia documentada só ocorreu entre os anos de 1981 a 1982, desde então se registra os dados sobre essa doença que vem ocorrendo de forma continuada (DIRETRIZES NACIONAIS PARA A PREVENÇÃO E CONTROLE DE EPIDEMIA DE DENGUE, 2009).

Segundo o Manual de Normas Técnicas, desenvolvido pela Fundação Nacional de Saúde (FUNASA), a dengue pode ser conceituada como uma doença febril aguda, onde o individuo apresenta em seu quadro patológico dores musculares e articulares intensas A transmissão da doença se faz pela picada da fêmea do mosquito Aedes aegypti, no ciclo ser humano - mosquito - ser humano (INSTRUÇÕES PARA PESSOAL DE COMBATE AO VETOR, 2001). A transmissão do ser humano para o mosquito ocorre enquanto houver presença de infecção no homem. Este período começa um dia antes do aparecimento dos sintomas e vai até o $6^{\circ}$ dia da doença. Após a ingestão do sangue humano infectado, o mosquito está apto a transmitir o vírus depois de 8 a 12 dias (período de incubação extrínseca) e assim permanece durante toda sua vida (MANUAL TÉCNICO PADRONIZAÇÃO DAS AÇÕES PARA CONTROLE VETORIAL DA DENGUE, 2009). No homem, o período entre a picada e o aparecimento de sintomas da doença pode variar de 3 a 15 dias sendo, em média, de 5 a 6 dias.

Os fatores condicionantes da expansão da dengue no Brasil referem-se, em grande parte, ao modelo econômico implantado, caracterizado pelo crescimento desordenado dos centros urbanos. Mais de $80 \%$ da população brasileira reside nas cidades, as quais apresentam importantes lacunas no setor de infraestrutura, tais como dificuldades para garantir o abastecimento regular e continuo de água, coleta e destino adequado dos resíduos sólidos. (MANUAL TÉCNICO PADRONIZAÇÃO DAS AÇÕES PARA CONTROLE VETORIAL DA DENGUE, 2009). Essa conjuntura aliada ao clima tropical do Brasil proporciona ambiente favorável à proliferação das epidemias de dengue.

Nessa seção vamos expor as características da doença, a forma de transmissão, os seus sintomas, e também expor alguns conceitos que norteiam as Diretrizes Nacionais para 
Prevenção e Controle de Epidemias de Dengue que é "um documento desenvolvido com o intuito de organizar, orientar, facilitar, agilizar e uniformizar as ações necessárias ao combate a dengue entre os integrantes do Sistema Único de Saúde" (MANUAL TÉCNICO PADRONIZAÇÃO DAS AÇÕES PARA CONTROLE VETORIAL DA DENGUE, 2009).

\section{ESTADO DA ARTE}

Diversos trabalhos foram encontrados na literatura que tratam do tema tratado nesse trabalho. Em Paula \& Deppe (2005) foi desenvolvido um Sistema de Informações Geográficas para o monitoramento e controle da dengue no estado do Paraná, tendo por objetivo unificar os dados dos seguintes sistemas: Sistema de Informações de Agravos Notificados (SINAN) e o Sistema de Informações da Febre Amarela e Dengue (SISFAD), ambos são utilizados pela Secretaria de Saúde daquele Estado. Segundo os autores os sistemas citados anteriormente são "desintegrados e não permitem a realização de qualquer tipo de análise espacial” (PAULA; DEPPE, 2005). Sendo assim, o sistema proposto por eles tem por características o suporte a um banco de dados robusto para auxiliar a espacialização dos casos de dengue no Estado do Paraná, permitindo a realização da sobreposição da incidência dos vetores da doença e da infestação dos seus vetores.

Nessa mesma linha podemos citar outra contribuição importante em Nascimento \& Petta (2011). O objetivo deste trabalho consiste na espacialização anual dos casos de dengue, entre os anos de 2000 e 2004, no Estado do Rio Grande do Norte utilizando técnicas de geoprocessamento. Para realização deste trabalho os autores elaboraram um Banco de Dados Georreferenciado (BDG) com informações dos números de casos de dengue em cada município do Estado. Esses dados foram "cedidos pela Secretaria de Saúde do Rio Grande do Norte, oriundas do Departamento de Informática do Sistema Único de Saúde do Brasil (DATASUS) e do SINAM" (NASCIMENTO; PETTA, 2011). Após o processamento dos dados, o próximo passo do trabalho foi à utilização de técnicas disponíveis no Sistema de Informação Georeferenciado, com o objetivo simular a tendência de risco da concentração e da dispersão da epidemia. Essa analise mostrou um aumento da epidemia a partir de 2001 e uma diminuição considerável no ano 2004 no Estado do Rio Grande do Norte.

Outra contribuição importante está no trabalho de Farias \& Candeias (2010) que propunham as medidas iniciais necessárias para o desenvolvimento de um Sistema de Informações Geográficas (SIG) para navegação de informações sobre a dengue. O projeto foi desenvolvido como parte integrante do Projeto do Sistema de Aquisição e Processamento de Imagens de Ovitrampas (SAPIO) da Universidade Federal de Pernambuco. Para atingir o objetivo, os autores utilizaram uma base de dados espaciais com a geração de cartas temáticas. Esses dados foram criados a partir de informações recolhidas das etapas anteriores do projeto, e por pesquisas desenvolvidas durante o trabalho. Entre os dados espaciais foram destacados informações sobre casos de dengue no período de 2000 a 2008, informações unidades de saúde existentes e informações socioeconômicas referentes aos bairros do município de Recife e a relação entre os mesmos. Para os autores o sistema desenvolvido por eles "permitirá à realização das análises espaciais necessárias a identificação do ambiente propício ao aparecimento e crescimento da dengue" (FARIAS; CANDEIAS, 2010). 
Nos trabalhos citados anteriormente, os autores se preocuparam com a incidência da dengue analisando dados dos casos notificados. Em Silva et al (2012) o foco é outro. O objetivo é a monitorização da contagem dos ovos do mosquito, e logo após a geração de gráficos e mapas georenfenciados. O Projeto desenvolvido consistiu em quatro etapas, divididos em coletagem, digitalização, contagem semiautomática e o Sistema de Informação Georeferenciado. Foi utilizado um sistema que digitaliza as imagens das paletas das ovitrampas, que são armadilhas com lavircida que atraem o mosquito, o Sistema de Digitalização de Palhetas (SDP) digitaliza os dados e envia para um servidor. As informações geradas através da contagem dos ovos podem ser visualizadas através de gráficos e mapas. Segundo os autores, o sistema tem por objetivo informar ao governo o local do foco de dengue para que as ações sejam executadas contra a infestação mais rapidamente, evitando um surto da doença (SILVA et al 2012).

\section{TECNOLOGIAS UTILIZADAS PARA A CONSTRUÇÃO DO SISTEMA}

Para o desenvolvimento do sistema exposto neste artigo, foram utilizadas as seguintes tecnologias: um framework de desenvolvimento web, o banco de dados PostgreSQL, a plataforma de dispositivos móveis Android, o Padrão REST, o Sistema de Posicionamento Global (GPS), a API do Google Maps.

Framework é "uma coleção de bibliotecas" (CARNEIRO JÚNIOR; BARAZI, 2011), que oferece ferramentas que facilitam os processos mais comuns no desenvolvimento de uma aplicação. Tornaram-se populares na medida em que agilizam o processo de desenvolvimento. O Ruby on Rais é um framework web completo, de código aberto, multiplataforma, possuindo uma camada de abstração para banco de dados. Uma das suas características marcantes é a presença de um conjunto considerável de padrões, e suporte a organização de arquivos que facilita no processo de implementação da aplicação. (CARNEIRO JÚNIOR; BARAZI, 2011).

Os dados utilizados são armazenados em um banco de dados PostgreSQL, que um Sistema de Gerenciamento de Banco de Dados Relacional (SGBDR) escolhido por oferecer integridade de dados transacional, alta disponibilidade e suporte a integração com linguagens de programação como o Java.

A Plataforma Android foi escolhida para o desenvolvimento do aplicativo móvel por ser uma plataforma completa, que inclui Sistema Operacional (S.O.), middleware e aplicações, possuindo também, diversos componentes com uma variada disponibilidade de bibliotecas e interface gráfica e ao qual facilita o desenvolvimento de aplicativos, utilizando a linguagem Java (LECHETA, 2009). Adicionalmente, a plataforma possui suporte a interfaces faladas e de síntese de voz, permitindo desenvolver aplicações com suporte à acessibilidade a deficientes visuais.

O estilo arquitetural REST é um conjunto de especificações utilizado sobre o protocolo HTTP (Hypertext Transfer Protocol) que define um padrão para a comunicação de webservices, visando escalabilidade e flexibilidade no formato das mensagens (MORO et al. 2009). É utilizado na implementação do WebService que permite interação do servidor núcleo do e-Guia com outras partes do sistema.

O Sistema de Posicionamento Global (GPS) é um sistema de posicionamento por satélite que envia via rádio para um aparelho receptor móvel, a posição de um receptor na superfície da Terra (BERNADI; LANDIM, 2002). O uso do GPS tem como função obter a 
localização exata do Agente de Endemias na hora do preenchimento do Registro de Trabalho de Campo, obtendo informações como latitude e longitude, que logo após serão geolocalizadas em um mapa com o auxilio da API do Google Maps.

A API do Google Maps pode ser conceituada como uma camada de software que é utilizada com o auxilio da Linguagem de Programação JavaScript que possibilita a aplicação ter acesso a estrutura de Maps e serviços oferecidos pela Google (GOOGLE DEVELOPER, 2013). Cada elemento do mapa na API é uma espécie de classe na orientação a objetos, onde podem ser instanciados, modificados, habilitados ou desabilitados, por meio de construtores, propriedades e métodos. Pode-se, por exemplo, habilitar ou não o zoom nos mapas, habilitar ou não as opções de visualização dos mapas por satélite ou terreno, criar ícones próprios em substituição aos ícones padrões.

\section{VISÃO GERAL DA ARQUITETURA DO SISTEMA}

A arquitetura de software pode ser conceituada como à organização de um sistema incorporada em seus componentes e em seus relacionamentos com o ambiente (IEEE/1471-2000). Sendo a forma de como o sistema vai interagir com os seus elementos internos e com os elementos externos a ele.

A arquitetura em referência desse sistema foi desenvolvida de forma modularizada e distribuída onde cada fluxo de execução é responsável por descrever uma parte importante do sistema, podendo ser representada pela Figura 1.

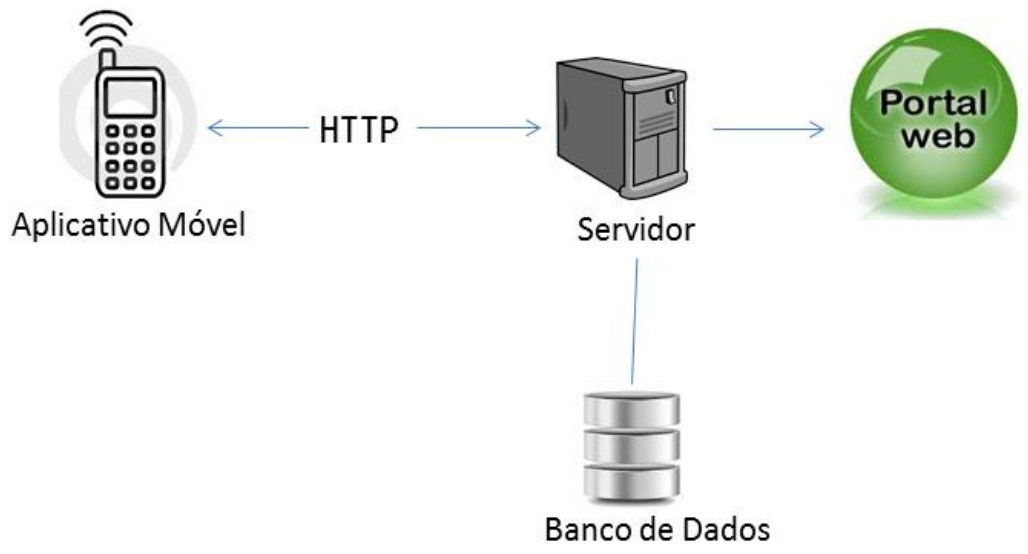

Figura 1 - Arquitetura do Sistema

O estilo arquitetural é o Cliente-Servidor que é um modelo de arquitetura que se baseia em um método de distribuição de aplicações computacionais através de multiplataformas, onde as aplicações são divididas entre o servidor de acesso e uma central de dados, que podem comunica-se com vários clientes, com todo o processamento realizado de forma distribuída (DESCHAMPS, et al. 1998). Esse processamento distribuído, também denominado de processamento concorrente utiliza-se do mecanismo de passagem de mensagens para a comunicação entre processos, ao qual possibilita a integração entre os módulos. O sistema é composto pelo Módulo do Agente de Endemias, pelo Módulo Portal e pelo Módulo Servidor.

O Módulo do Agente de Endemias é um aplicativo móvel onde são registradas as informações da atividade de Campo do Agente. A função do aplicativo móvel é a emissão e recepção da informação a partir de um determinado local (LEMOS, 2007). Sendo assim, 
as informações sobre os focos são coletados através dos dispositivos móveis e enviados ao servidor como o objetivo de obter a localização exata da visita realizada pelo Agente de Endemias.

Esses dados após coletados são enviados para o Módulo Servidor, que recebe via protocolo HTTP, os dados das visitas, que logo após essas informações podem ser consultadas pelo Módulo Portal. A representação da comunicação entre os módulos do sistema pode ser verificada pela Figura 2, onde fica evidente a distribuição da aplicação.

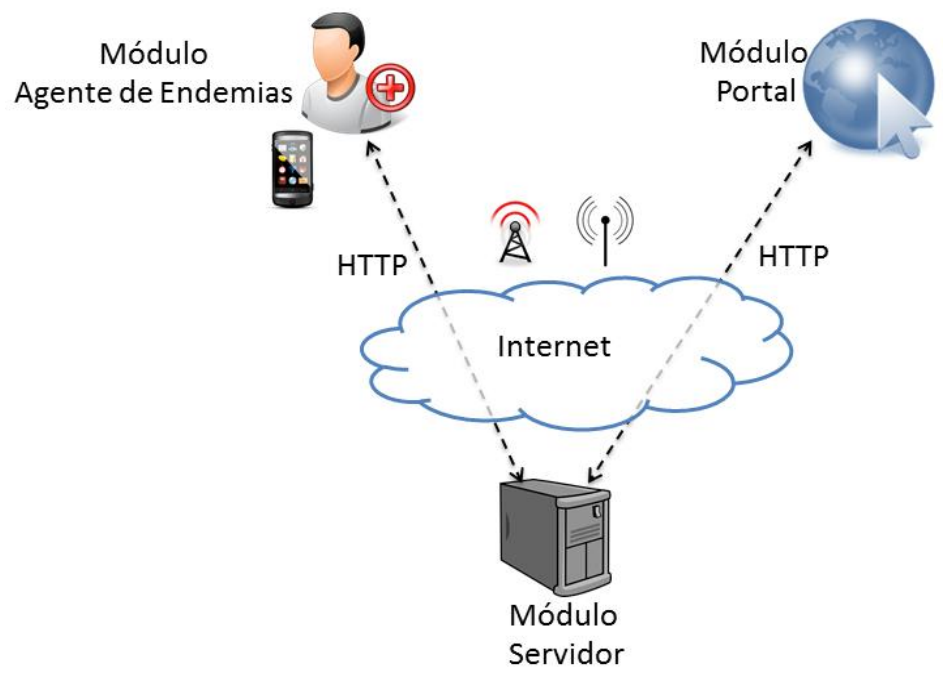

Figura 2 - Representação da Comunicação entre os Módulos do Sistema

Desta forma, os módulos são executados sobre diferentes plataformas e comunicando-se mediante a utilização de componentes responsáveis por gerenciar as chamadas remotas, o que possibilita uma maior eficiência, transparência, escalabilidade e suporte a recursos compartilhados do sistema.

\section{MÓDULO DO AGENTE DE ENDEMIAS}

A visita domiciliar é uma atividade fundamental do Agente de Endemias que tem por objetivo a verificação da presença de criadouros, a orientação aos residentes sobre a eliminação dos mesmos; e como também, a promoção de medidas preventivas, e a identificação de foco e tratamento focalizado no mosquito, sendo a parte central das atividades de campo (DIRETRIZES NACIONAIS PARA A PREVENÇÃO E CONTROLE DE EPIDEMIA DE DENGUE, 2009).

A parte central do Registro das Atividades de Campo é o Registro Diário do Serviço Anti-vetorial. Esse registro consiste em um formulário de coleta sobre a Visita Domiciliar do Agente de Endemias denominado Registro Diário do Serviço Anti-vetorial. No sistema proposto, este Registro foi dividido em três categorias: Localização, Informações sobre a Visita e Tratamento.

Na categoria Localização são inseridas informações sobre a localização do imóvel tais como: município, nome localidade, zona, número do quarteirão, ciclo nem sempre corresponde ao ano corrente. Essa informação é de extrema importância para o levantamento estatístico sobre a doença. 
Na categoria Informações sobre a visita, são selecionadas informações gerais em relação à visita, como o tipo de imóvel que pode ser residencial, comercial, terreno baldio e outros; e também, o agente pode informar o número de depósitos que foram eliminados, que consiste na quantidade de recipientes com a presença do mosquito. Na categoria Tratamento são preenchidas as informações sobre o tratamento do foco tais como o tipo de lavircida usado, a quantidade de gotas, o número de excesso de gotas, e o número de depósitos que foram tratados.

Para o desenvolvimento da interface do formulário, optou-se por usar o modelo de abas, com o objetivo de atender os seguintes requisitos: facilidade de aprendizado e, prevenção e redução de erros buscando assim, obter uma usabilidade satisfatória para o usuário. Outro recurso importante foi desenvolvido buscando atender a esse requisito, foi à possibilidade do usuário deslizar sobre as abas sem perder as informações por ele preenchidas, o que reduz o número de clicks e obtêm-se agilidade na hora do preenchimento das informações. A Figura 3 dar uma visão geral do aplicativo móvel desenvolvido.

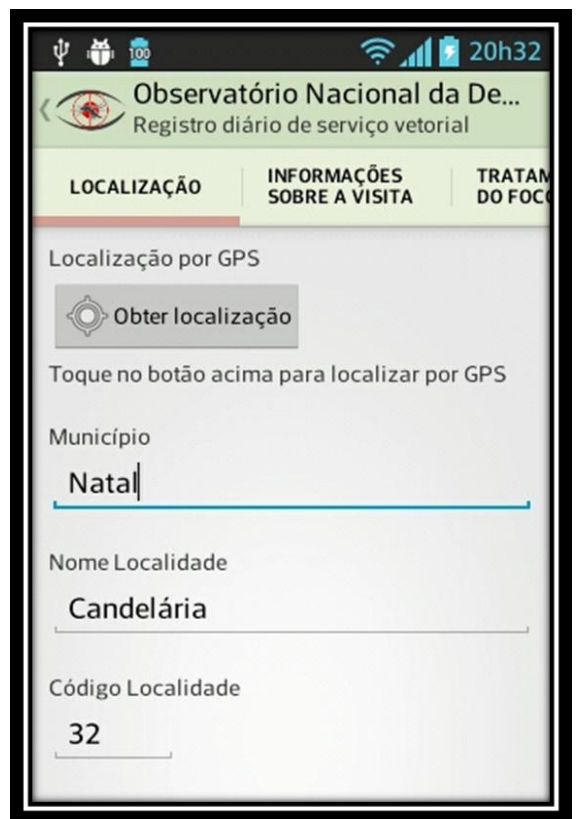

Figura 3 - Visão Geral do Aplicativo Móvel

No formulário foi implementado um recurso importante, que é central para o objetivo geral do sistema, que é a localização do Agente de Endemias através do GPS que possibilita que sejam coletadas as informações sobre latitude e longitude, e é representada pela Figura 4. Essas coordenadas têm por objetivo possibilitar a geolocalização da visita no mapa, que logo serão visualizadas no portal.

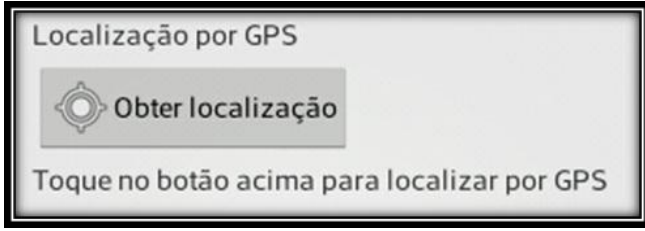

Figura 4 - Botão para obter a localização do Agente de Endemias

Para desenvolver esta funcionalidade foi utilizado um gerenciador de localização para chamar o GPS, que no Android é representado pela classe LocationManager. Assim, quando o usuário clica em Obter Localização o evento é acionado, apenas uma vez, e a 
localização é obtida. No entanto, se o GPS do aparelho estiver desligado é emitido um aviso, representado pela Figura 5, perguntando se o usuário deseja habilitar as configurações, Se o usuário clicar em Ok, um serviço é acionado em segundo plano fazendo com que a aplicação chame as configurações do aparelho, onde o GPS vai ser acionado.

\begin{tabular}{|c|c|}
\hline \multicolumn{2}{|l|}{ Aviso } \\
\hline \multicolumn{2}{|c|}{$\begin{array}{l}\text { O GPS do dispositivo está } \\
\text { desligado. Você deseja abrir as } \\
\text { configurações para habilitá-lo? }\end{array}$} \\
\hline Cancelar & OK \\
\hline
\end{tabular}

Figura 5 - Aviso GPS desligado

\section{MÓDULO PORTAL}

O Portal Web no trabalho proposto tem por objetivo disponibilizar a população e para o gestor os dados sobre a dengue coletados durante a atividade de campo, a Figura 6 ilustra a página inicial do Observatório. A parte púbica é composta por uma seção que agrega feed de noticias de todo o país, sobre o que esta sendo publicado sobre a dengue, além de uma página de contato. E um local onde pode ser feito pela população uma pesquisa sobre as visitas realizadas pelos agentes.

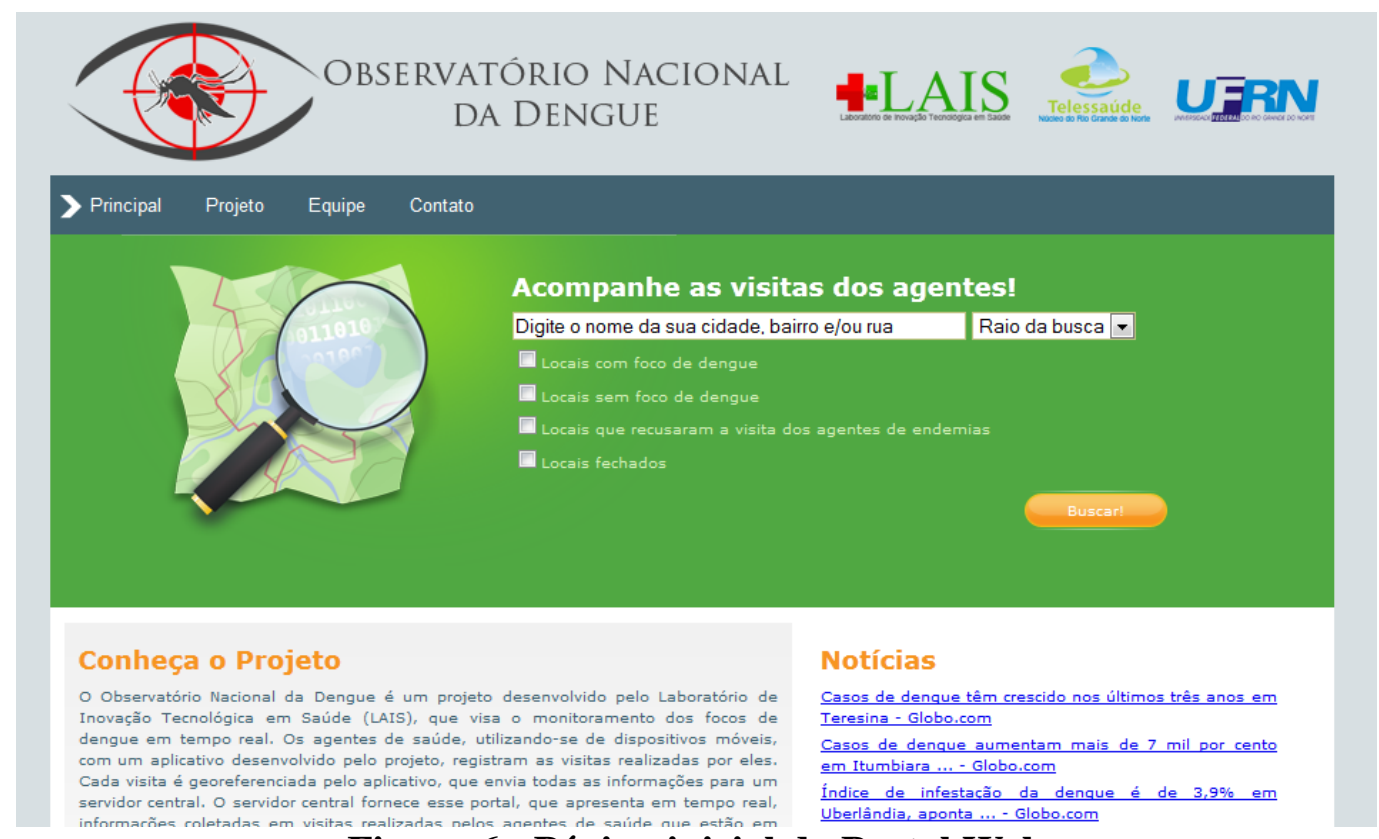

Figura 6 - Página inicial do Portal Web

A funcionalidade mais importante do Portal é a busca sobre os dados a respeito dos focos. Nela o usuário pode escolher o raio de busca, as opções de locais que podem ser: com foco, sem foco, locais que recusaram a visita dos agentes, locais fechados e todos os locais. Logo após digita o nome da cidade, bairro ou rua e clicar no botão, a busca é realizada e será inserida em um mapa representado pela Figura 7. Essa inserção no mapa só é possível porque no aplicativo móvel é coletada, via GPS, a latitude e longitude da Rua que está sendo realizada a visita. Essa informação é salva em um banco de dados. 
O mapa é iniciado com o auxilio da API do Google Maps, para isso foi criando um objeto Map options, passando todos os parâmetros devem ser expostos no mapa. Foi desenvolvido um método, por meio de uma função JavaScript, para converter o objeto que representa a situação ou a pendência da visita em um marcador do Google Maps, e quando o usuário coloca o mouse em cima desse marcador é mostrado um InfoWindow que exibe os detalhes da visita realizada tais o horário do registro, se havia foco ou não e o número daquela visita. Após a pesquisa um pequeno relatório é exibido ao lado do mapa com uma síntese dos dados, levando em consideração os parâmetros usados.

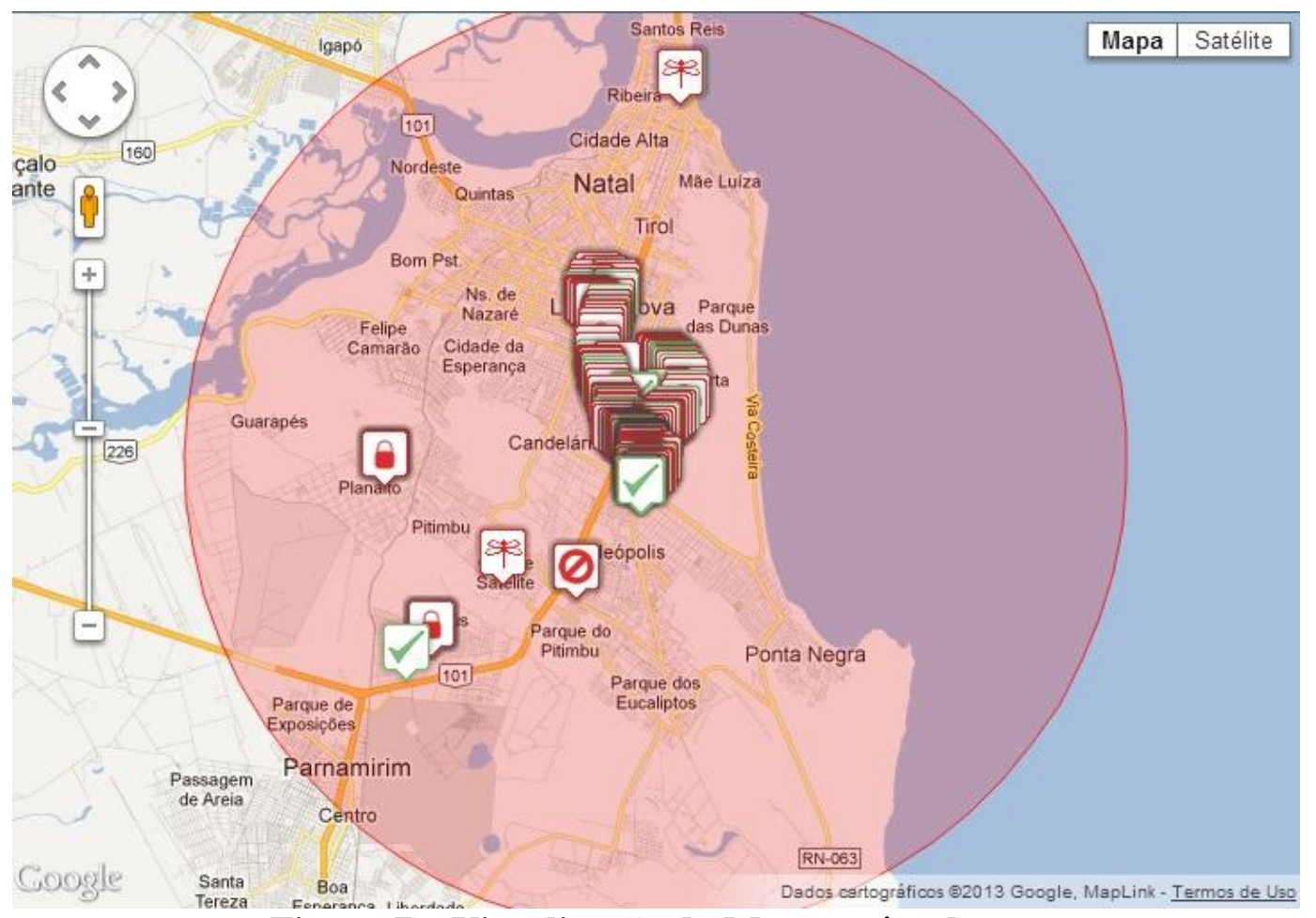

Figura 7 - Visualização do Mapa após a busca

\section{EXPERIMENTOS}

Com o objetivo de validar a arquitetura foram realizados experimentos que foram divididos em duas categorias: os iniciais e o teste real com o Agente de Endemias.

Os experimentos iniciais foram realizados pela equipe de desenvolvimento do projeto, onde foi validada a primeira versão do sistema. Esse teste tinha por objetivo verificar se as tecnologias necessárias para o funcionamento do sistema tais como as redes de comunicação sem fio, e o envio das informações via HTTP funcionariam de forma satisfatória, para isso foram simuladas visitas em vários pontos da cidade do Natal. Sendo assim, o objetivo desses testes era a validação da arquitetura construída.

O segundo experimento, contou com a presença do Agente de Saúde, o objetivo era testar a usabilidade do aplicativo móvel. Para este experimento dois parâmetros foram seguidos, primeiro verificou-se a interface com relação à usabilidade; e segundo, a Interação Homem-Máquina, onde foi avaliado o feedback fornecido pelo sistema, nos quesitos clareza e entendimento do sistema por parte do usuário. 
Com relação à usabilidade da interface o objetivo era comprovar se o usuário seria capaz de: identificar as ações necessárias e realizar o preenchimento do formulário de forma correta, e seguir com precisão o fluxo realizado pelo sistema.

Com relação ao feedback o objetivo era testar se o sistema seria capaz de interagir com o usuário nas seguintes situações: o usuário se autenticaria no sistema, e o usuário enviaria o formulário sem preencher todos os campos.

\section{RESULTADOS E DISCUSSÕES}

A realização dos experimentos comprovou a viabilidade do sistema desenvolvido, uma vez que o mesmo funcionou como o esperado. Os testes iniciais validaram a arquitetura desenvolvida, onde foram simuladas várias visitas em torno de um Bairro de Natal. Esses dados podem ser pesquisados no Portal Web, e simulando uma pesquisa nesse portal em um raio de $10 \mathrm{~km}$ do bairro podemos verificar a seguinte situação das visitas, de acordo com a Tabela 1.

\begin{tabular}{|l|l|l|l|}
\hline Com foco & Sem foco & Visita Recusada & Locais Fechados \\
\hline 54 & 48 & 39 & 32 \\
\hline
\end{tabular}

Tabela 1 - Simulação da situação das visitas realizadas

O teste com o usuário demonstrou uma resposta satisfatória do sistema. Com relação ao primeiro parâmetro observado que foi a usabilidade, o usuário foi capaz de compreender as ações necessárias e realizar o preenchimento do formulário de forma correta, e seguir com precisão o fluxo realizado pelo sistema.

Com relação ao feedback verificou-se que o sistema foi capaz de interagir com o usuário em duas situações. Na primeira situação o usuário se autenticaria no sistema, no entanto o formulário não é preenchido, mas, clica no botão de voltar do aparelho. Nessa situação o sistema foi capaz de exibir o alerta para o qual estava programado, que foi: "Você não enviou o formulário. Tem certeza de que quer sair?". Este alerta está representado pela Figura 8.

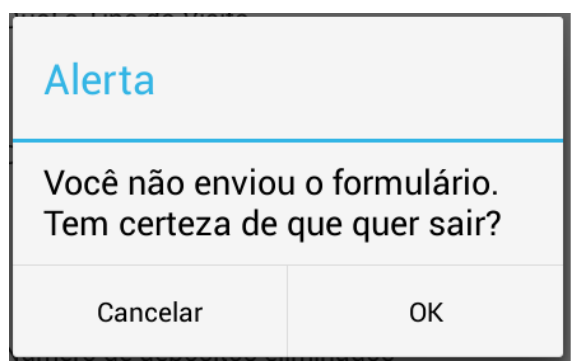

Figura 8 - Alerta do sistema sobre o envio do formulário

Na segunda situação o usuário enviou o formulário sem que todos os dados fossem preenchidos. Nessa situação o sistema foi capaz de voltar ao estado que se encontrava antes do envio, e destacar apenas os campos que não foram preenchidos pelo usuário, conforme mostra a Figura 10. Assim, todos os campos não preenchidos foram destacados em vermelho, para que o usuário terminasse a execução da tarefa. 


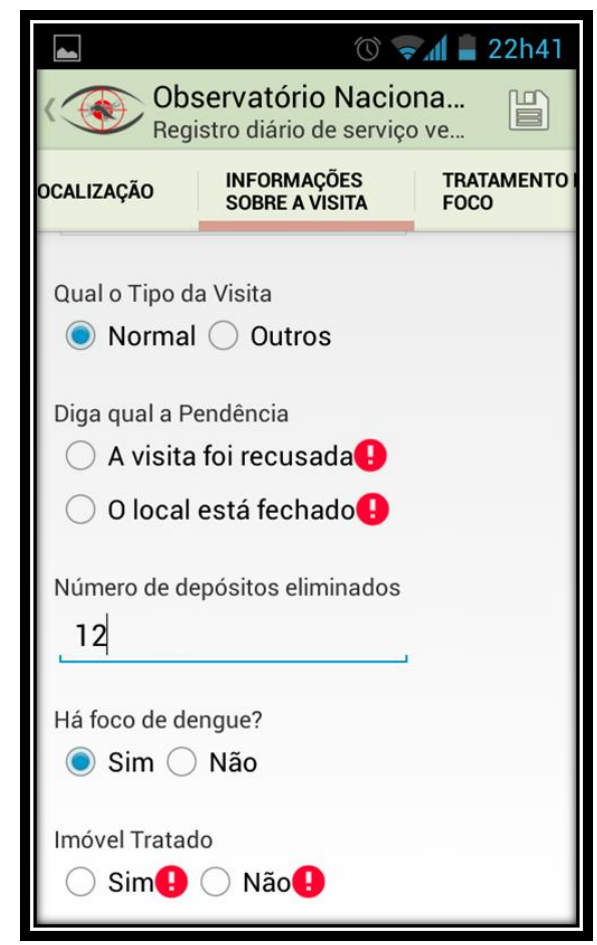

Figura 9 - Erro de informações não preenchidas

Com esse trabalho foi possível desenvolver uma ferramenta para auxiliar na operacionalização das atividades do combate a dengue, na medida em que o aplicativo móvel mostrou-se uma ferramenta viável para a informatização das ações dos Agentes de Endemias.

Outro aspecto importante a ser considerado é a gestão estratégica da informação na area da saúde, que tem que ser observada dentro de sua dimensão política e estratégica, onde o grande desafio não é apenas a divulgação dos dados, e sim, a sua decodificação em uma linguagem que possa ser entendida, de forma a permitir que qualquer individuo possa se apropriar do conhecimento. Desta forma, esse sistema mostrou-se como uma importante ferramenta de divulgação sobre os dados da dengue, utilizando para tal uma linguagem visual como um mapa.

\section{REFERÊNCIAS BIBLIOGRÁFICAS}

1. ANDROID. Android App Framework. Disponível em http://developer.android.com/about/versions/index.html. Acesso em: março/2013

2. ARAÚJO, B. G. de. Modelo Arquitetural de Comunicação para Monitoramento de Pacientes Baseado em Middleware, Computação Móvel e Ubíqua. 2010. 72 f. Dissertação (Mestrado em Engenharia Elétrica e Computação) - Universidade Federal do Rio Grande do Norte, Natal, 2010.

3. BERNARDI, J.V.E ; LANDIM, P.M.B. Aplicação do Sistema de Posicionamento Global (GPS) na coleta de dados. DGA, IGCE, UNESP/Rio Claro, Lab. Geomatemática, Texto Didático 10, 31 pp. 2002. Disponível em http://www.rc.unesp.br/igce/aplicada/textodi.html. Acesso em: janeiro/2013. 
4. DEPARTAMENTO DE CIÊNCIA E TECNOLOGIA, Secretaria de Ciência. Tecnologia e Insumos Estratégicos, Ministério da Saúde. Tecnologia. Rede Dengue: inovação da abordagem e da gestão em pesquisa à saúde. v. 44, n. 6 , p. 1159-1163, 2010.

5. DEPARTAMENTO DE VIGILÂNCIA EPIDEMIOLÓGICA. Ministério da Saúde. Diretrizes Nacionais para a Prevenção e Controle de Epidemias de Dengue. Brasília, 2009. Disponível em: http://www.matrizes.usp.br/index.php/matrizes/article/view/29/43. Acesso em: fevereiro /2013.

6. DONALÍSIO, M. R; GLASSER, C. M. Vigilância Entomológica e Controle de Vetores do Dengue. Rev. Bras. Epidemiol, v. 5, p. 259-272, 2002.

7. FARIAS, E. S. de; CANDEIAS, A. L. B. Desenvolvimento de um Sistema de informações Geográficas para Navegação de Informações sobre a Dengue. III Simpósio Brasileiro de Ciências Geodésicas e Tecnologias da Geoinformação. Recife - PE, 27-30 de Julho de 2010, p. 001 de 003.

8. GIOVAnElla, L. Atenção Primária à Saúde. In: Políticas e Sistema de Saúde no Brasil. Rio de Janeiro: Editora FIOCRUZ, 2008. Parte IV, pag. 575 a 626

9. Introdução a Banco de Dados. Disponível em: http://www.ime.usp.br/ jef/apostila.pdf.

10. KUROSE, J. F; ROSS, K. W. Redes de Computadores e a Internet: uma abordagem top-down. Ed. Pearson Education, 5 ed.

11. LECHETA, R. R. Google Android: Aprenda a criar aplicações para dispositivos móveis com o Android SDK. 2 ed. São Paulo, Novatec, 2010.

12. LEMOS, A. Cidade e mobilidade. Telefones celulares, funções pós-massivas e territórios informacionais. Matrizes, v1, n1, 2007.

13. M. G. N. M. da Silva, V. C. Casé, M. A. B. Rodrigues e R. E. de Araújo. Sistema de Informação Geográfica Para o Auxílio ao Controle da Dengue. XXIII Congresso Brasileiro em Engenharia Biomédica - CBEB, 2012.

14. MANUAL DE NORMAS TÉCNICAS. Instruções para Pessoal de Combate ao Vetor. Fundação Nacional da Saúde, Brasília - DF, 2001.

15. MANUAL DE RECONHECIMENTO GEOGRÁFICO. Secretaria de Estado da Saúde. Palmas - TO, 2007.

16. MANUAL TÉCNICO PADRONIZAÇÃO DAS AÇÕES PARA CONTROLE VETORIAL DA DENGUE. Secretaria Municipal de Saúde - SMSA. Belo Horizonte, 2009.

17. MENEGOTTO, A. B.; MIERLO, F. A linguagem Ruby. Disponível em http://menegotto.com/articles/ruby.pdf . Acesso em: janeiro/2013. 
18. MORO, T. D.; DORNELES, C.; REBONATTO, M. T. Web Services WS-* versus Web Services REST. Revista de Iniciação Cientifica. v 11, n.1. 2011. Disponível em http://seer.ufrgs.br/reic/article/view/22140 Acesso em: janeiro/ 2013

19. NASCIMENTO, P. S. de R.; PETTA, R. A. Uso de Sistema de Informação Geográfica na dispersão de casos de dengue no Estado do Rio Grande do Norte. Anais XV Simpósio Brasileiro de Sensoriamento Remoto - SBSR, Curitiba, PR, Brasil, 30 de abril a 05 de maio de 2011, INPE p.8421.

20. PAULA, E. V.; DEPPE, F. SIG-Dengue: Sistema de Informações Geográficas para o monitoramento e controle da dengue no estado do Paraná. Anais XII Simpósio Brasileiro de Sensoriamento Remoto, Goiânia, Brasil, 16-21 abril 2005, INPE, p. 2309-2311.

21. SBIS, Sociedade Brasileira de Informática na Saúde. O que é informática na Saúde. 2013. Disponível em http://www.sbis.org.br/indexframe.html. Acesso em: janeiro/2013.

22. TAKAI, O. K.; ITALIANO, I. C; FERREIRA, J E.

23. TOBALDINI, R. G. Arquitetura REST: um estudo de sua implementação em linguagens de programação. 2007. Monografia. UNIVERSIDADE FEDERAL DE SANTA CATARINA, Florianópolis, 2006.

24. VOTANO, JR; PARHAM, M; HALL, LH. Arquitetura AAA em Sistema Web Baseados em REST. p. 1-7, 2004. Disponível em: http://onlinelibrary.wiley.com/doi/10.1002/cbdv.200490137/abstract. Acesso em: janeiro/2013. 